\title{
Estudio de concordancia entre el diagnóstico dínico y el diagnóstico anatomopatológico de lesiones dermatológicas en A tención Primaria
}

\author{
A. Gallego Ruiz, F. J. Peces Moratein, P. Elviro García², C. Sierra Garrido ${ }^{3}$ \\ MIR de $3^{0}$ año de MF y $C^{2}{ }^{2}$ Médico de Familia. Tutor de MIR de MF y $C^{2}{ }^{2}$ M édico \\ Especialista en Mediana Interna. ${ }^{3}$ M édico de Familia. Centro de Salud Embajadores I. \\ Área 11 INSALUD. Madrid
}

\section{RESUMEN}

Pretendemos estudiar el grado de concordancia, entre el diagnóstico clínico y el diagnóstico anato mopatológico, de las lesiones dermatológicas re mitidas desde cinco Centros de Salud del Área 11 de Madrid. Se trata de un estudio descriptivo en el cual examinamos la totalidad de lesiones dermato lógicas remitidas desde los mismos al Servicio de Anatomía Patológica del Hospital 12 de Octubre de Madrid, entre agosto de 1997 y febrero de 2002.

Se han analizado 203 lesiones dermatológicas procedentes de 199 pacientes ( 79 varones y 120 mujeres), con un intervalo de edad entre los 13 y 88 años y una media de 48,4 años. Los diagnósti cos clínicos han sido, fundamentalmente, pólipo fi broepitelial (67 casos), nevus melanocítico (45), y verruga vulgar (33). La concordancia global entre el diagnóstico clínico y el anatomopatológico ha resultado del $64 \%$. Se han hallado 4 casos $(1,97 \%)$ de epitelioma.

Creemos que la concordancia global es satisfac toria, y esto nos permite continuar desarrollando la cirugía menor en los Centros de Salud, en los términos establecidos en el correspondiente proto colo de Atención Primaria.

Palabras clave: Cirugía menor. Atención Prima ria. Médicos de familia.
Concordance study between clinical diagnosis and anatomical pathology diagnosis in cutaneous le sions in Primary Health Care centres

\section{ABSTRACT}

We try to study the concordance between clinical diagnosis and anatomical pathology diagnosis in cutaneous lesions in patients who come from five Primary Health Care centres of the Area 11. It is a descriptive study in which we examine all cuta neous lesions from five centers and then we send this examination to the Pathology Service of the 12 de Octubre Hospital, between August 1997 and Fe bruary 2002.

There were studied 203 cutaneous lesions obtai ned from 199 patients ( 79 male and 120 female), aged between 13 and 88 years (mean age 48.4 years old). Essentially, presurgical diagnosis were fibroepithelial papiloma (67 events), nevocellular nevi (45) and viral wart (33). There was $64 \%$ glo bal agreement between clinical diagnosis and his topathological diagnosis. There were found 4 skin cancer $(1.97 \%)$.

We believe that there is a satisfactory concor dance and this allow us to practice minor surgery in Primary Health Care centres as it is established in the Primary Health Care protocol.

Key words: Minor surgery. Primary Health Care. General practitioners. 


\section{INTRODUCCIÓN}

La cirugía menor (CM) que se realiza en los Centros de Salud (CS) supone un conjunto de procedimientos sencillos, de corta duración, sobre tejidos superficiales, fácilmente accesibles y donde existe un riesgo bajo y escasas posibilidades de complicaciones postquirúrgicas. Se trata de una prestación incluida en el programa de formación de residentes de Medicina de Familia, y en la Cartera de Servicios de Atención Primaria (AP) desde 1999, y cada año se está incrementando el número de médicos de familia, que toman la decisión de actuar sobre las lesiones susceptibles de tratamiento quirúrgico a este nivel, como ocurre con los abscesos cutáneos, fibromas blandos, nevus melanocíticos, queratosis actínicas y seborreicas, quistes epidérmicos y mucoides, verrugas vulgares, lesiones ungueales, etc., derivando a los Servicios de Dermatología o Cirugía aquellas lesiones sospechosas de malignidad o que no cumplen criterios de inclusión en el Protocolo'.

En cualquier caso el número de cirugías realizadas depende, inevitablemente, de la presión asistencial en AP y Atención Especializada (AE) y de la formación y motivación de los médicos de familia. En el año 1997 se realizaron alrededor de 5.000 intervenciones en los CS del INSALUD, y en el 2000 la cifra ascendió aproximadamente a 70.000, con un 3\% de complicaciones, frente al $6 \%$ de los procedimientos quirúrgicos llevados a cabo en el ámbito hospitalario, debido -claro está- a la mayor complejidad de la patología tratada ${ }^{2}$. Por otra parte, la realización de CM en AP tiene una aceptación favorable entre los usuarios ${ }^{3}$.

\section{OBJETIVO}

Este trabajo pretende estudiar el grado de concordancia entre el diagnóstico clínico o prequirúrgico y el diagnóstico anatomopatológico posterior, considerado patrón oro - gold standard-, de 203 lesiones extirpadas en 5 CS del Área 11 de Salud de Madrid y, por otra parte, analizar los resultados de los distintos CS y los factores que podrían influir en los mismos. Lo justificamos por el progresivo incremento -citado previamente-, de cirugías realizadas en los CS y por la necesidad de evaluar la capacidad de diagnóstico clínico de las lesiones dermatológicas extirpadas en los CS del Área 11 de Madrid. Así, analizamos cómo se desarrolla en la práctica diaria una parte de la CM y aportamos información para mejorar -si es posible- la asistencia sanitaria.

\section{MATERIAL Y MÉTODO}

El Área 11 de Salud de la Comunidad Autónoma de Madrid tiene como principal hospital de referen- cia al Hospital Universitario 12 de Octubre, donde se remiten las muestras para estudio histológico y citológico procedentes de $39 \mathrm{CS}$, que corresponden a 47 equipos de Atención Primaria. De ellos se han elegido 5 CS que presentan mayor actividad en CM: CS Los Ángeles, CS Comillas, CS Embajadores, CS El Espinillo y CS Guayaba, que aportan el $15,76,41,87,16,75,5,91$ y $19,7 \%$ de piezas al conjunto de 203 muestras estudiadas (Tabla I). Las piezas para análisis se han enviado al Servicio de Anatomía Patológica del Hospital Universitario 12 de Octubre.

\section{Tabla I}

DESCRIPCIÓN DE LA POBLACIÓN ESTUDIADA

203 piezas procedentes de 199 pacientes de 5 Centros de Salud

$\begin{array}{lr}\text { C.S. Los Ángeles } & 15,76 \% \\ \text { C.S. Comillas } & 41,87 \% \\ \text { C.S. Embajadores } & 16,75 \% \\ \text { C.S. El Espinillo } & 5,91 \% \\ \text { C.S. Guayaba } & 19,70 \%\end{array}$

79 varones $(39,7 \%)$ y 120 mujeres $(60,3 \%)$

Edad media: 48,4 +/ - 19,13 años ( $x+/$-SD)

Límites de edad: 13 y 88 años

La población diana objetivo del estudio son la totalidad de cirugías en las cuales se solicitó análisis anatomopatológico, y fueron realizadas en dichos CS entre el 6 de agosto de 1997 y el 28 de febrero de 2002, dado que los datos más antiguos encontrados eran de aquellas fechas y se empezó a recopilar información en marzo de 2002. Es decir, se excluyeron la mayoría de cirugías realizadas, que fueron drenaje de abscesos, heridas e infiltraciones. Los mismos autores han recogido los datos de las cirugías, registrados bien en los libros de registro de CM en cada CS o bien en el programa informático implantado en AP (OMI). Se han recopilado 203 casos que se corresponden con 199 pacientes, de los cuales 79 eran varones $(39,7 \%)$ y 120 mujeres $(60,3 \%)$. La media de edad ha sido $48,4 \pm 19,13$ años $(x \pm S D)$, con unos límites de edad entre los 13 y 88 años. En 14 casos no figuraba la edad del paciente en la ficha de CM. Las variables analizadas son edad, sexo, diagnóstico clínico, diagnóstico anatomopatológico y CS donde se ha realizado la CM. Hemos definido, tanto el diagnóstico clínico como el anatomopatológico en seis categorías, que son las lesiones más frecuentemente tratadas en AP: pólipo fibroepitelial, nevus melanocítico, verruga vulgar, queratosis seborreica, quiste epidérmico y epitelioma, incluyendo en una séptima categoría, denominada otros, el resto 
de lesiones. El término concordancia ha sido definido de forma que sólo figurasen uno o dos diagnósticos clínicos y luego coincidiera con el diagnóstico anatomopatológico. En la mayoría de las piezas remitidas solamente existía un diagnóstico prequirúrgico. Se ha incluido toda la información en una base de datos excel y, después, se ha aplicado el test de concordancia kappa, por separado para las muestras de cada CS y para la totalidad de la población analizada.

\section{RESULTADOS}

Excluidos la mayoría de procesos realizados, que han sido abscesos, heridas, infiltraciones y extirpaciones en las que no se han enviado las piezas al Servicio de Anatomía Patológica, se analizaron 203 casos. Por edad y sexo, las intervenciones se agrupan en varones entre los 46 y 64 años y en mujeres entre 26 y 45 años. El número de intervenciones realizadas en mujeres menores de 45 años es, prácticamente, el doble que las efectuadas en varones de la misma edad y, tratándose de lesiones benignas, aunque no se pueda demostrar, probablemente esté influenciado por motivos cosméticos.

Las muestras remitidas presentaban, en orden decreciente, los siguientes diagnósticos: pólipo fibroepitelial (33\%), nevus melanocítico $(22,17 \%)$, verruga vulgar $(16,26 \%)$, quiste epidérmico $(12,81 \%)$, queratosis seborreica $(9,85)$ y epitelioma $(2,46 \%)$ (Fig. 1).

Por su parte, los resultados encontrados fueron, también en orden de mayor a menor: pólipo fibroepitelial $(31,03 \%)$, nevus melanocítico $(26,11 \%)$, verruga vulgar $(14,78 \%)$, quiste epidérmico $(9,85 \%)$, queratosis seborreica $(8,87)$ y epitelioma $(1,97 \%)$. En dos casos el diagnóstico final -inesperado- fue poroma ecrino, clasificado clínicamente como nevus melanocítico y granuloma; en tres ocasiones una lesión que, en el CS, impresio-

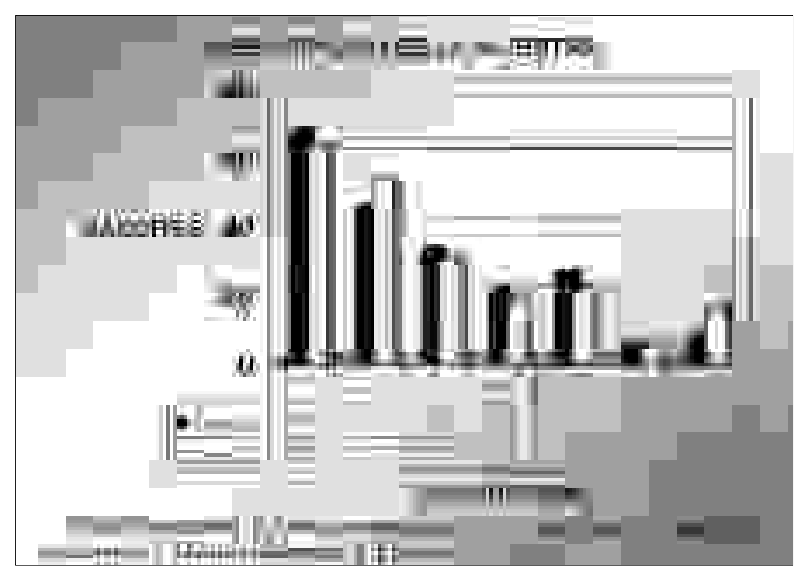

Figura 1 naba de quiste epidérmico resultó ser un pilomatrixoma, un tricoblastoma y un linfoma T CD 30+; y en seis casos más, la biopsia demostró vasculitis leucocitoclástica, molusco, poroqueratosis, queratosis actínica, hemangioma y angioqueratoma trombosado.

El índice kappa de concordancia global obtenido entre el diagnóstico clínico y el diagnóstico anatomopatológico posterior ha sido $\mathrm{k}=0,64$ con límites de confianza del $95 \%$ entre 0,55 y 0,72 , existiendo un grado de acuerdo similar entre los diferentes CS estudiados (Tabla II). Se trata de una concordancia similar a la hallada en otros estudios publicados por Guereña et $\mathrm{al}^{4}$ que afirman encontrar una coincidencia del $63 \%$, e inferior a los estudios de Arribas et al que publican unas cifras del $80 \%{ }^{5}$, Pérez Unanua et al que afirman $77,7 \%{ }^{6}$ y mayor al $30-45 \%$ que refiere Cox et $\mathrm{al}^{7}$.

\section{DISCUSIÓN}

Varios factores influyen en los diferentes valores del índice de concordancia kappa, obtenidos en otros tantos CS. Como apuntan Menárguez Puche et al $^{8}$ sería necesario aclarar cuál es el número adecuado de piezas que se deben remitir para biopsia para valorar la calidad de la CM realizada en AP, pero además es evidente que los resultados van a ser muy diferentes en función del comportamiento de cada médico de familia. En este sentido, en nuestra opinión, distinguimos tres actitudes: sólo enviar aquellas piezas en las cuales el diagnóstico clínico es más dudoso, de manera que la concordancia diagnóstica disminuye; remitir todas las piezas que se extirpen, independientemente de la dificultad en el diagnóstico clínico, obteniendo mayor concordancia; y un tercer comportamiento, incluido en realidad dentro de éste último, sería intervenir y enviar sólo aquellas lesiones en las que la seguridad del diagnóstico clínico es casi absoluta, asegurando el máximo de concordancia.

\begin{tabular}{|lcc|}
\multicolumn{1}{c}{ CONCORDANCIA POR CENTROS DE SALUD } \\
\hline & Índice kappa & $\begin{array}{c}\text { Intervalo } \\
\text { confianza 95\% }\end{array}$ \\
\hline C.S. Embajadores & 0,67 & $0,47-0,87$ \\
C.S. Comillas & 0,56 & $0,43-0,69$ \\
C.S. El Espinillo & 0,85 & $0,56-1$ \\
C.S. Los Ángeles & 0,57 & $0,34-0,81$ \\
C.S. Guayaba & 0,71 & $0,52-0,9$ \\
\hline Global & 0,64 & $0,55-0,72$ \\
\hline
\end{tabular}


Por otra parte, cada vez se utiliza más la crioterapia (Histofreezer ${ }^{\circledR}$ ) para el tratamiento de diferentes lesiones virales y otros procesos ${ }^{9}$ y esta técnica imposibilita la posterior realización de biopsia. Por todo ello, habría que definir las razones fundamentales para remitir las piezas a analizar y utilizar, sobre todo, criterios cualitativos, fundamentalmente la posibilidad o no de estar ante una lesión premaligna, para lo cual la solución incluiría mejorar la capacidad diagnóstica de los médicos de familia. En defensa de este aumento de la capacidad de diagnóstico en AP, realizando cursos de formación, rotaciones por los Servicios de Dermatología, etc., encontramos otro trabajo publicado ${ }^{10}$ que compara la concordancia diagnóstica global entre AP y AE, concluyendo que la misma es baja, resultado lógico pues estudia pacientes remitidos desde los CS a las consultas de Dermatología, que además precisaron estudio anatomopatológico, y se trata, por tanto, de pacientes de difícil diagnóstico clínico.

\section{AGRADECIMIENTOS}

Agradecemos la colaboración para facilitar la obtención de los datos necesarios para realizar este estudio al Dr. González-Valcárcel, Dr. Blanco, Dr. Revuelta, Dra. Bonaplata, Dra. Vargas-Machuca, Dra. Fernández Abad, Dr. Delgado Nicolás, Dr. Caballero, Dr. Martín-Calle, Dr. Garcés y Da Rosa Prieto Corchete, enfermera responsable de CM en el CS Embajadores, así como al Dr. Jesús Martín y Dr. Gómez de la Cámara por su ayuda para la aplicación de los programas informáticos.

\section{CORRESPONDENCIA:}

Francisco Javier Peces Morate

Centro de Salud Embajadores

C/ Cardenal Solís, 6

28012 Madrid

Tel.: 91-4671200

\section{Bibliografía}

1. Protocolo de Cirugía menor en Atención Primaria. Instituto Nacional de la Salud. Área 11. Madrid, 1998. p. 9, 23.

2. Taller dermatología quirúrgica para médicos de Atención Primaria. Area 11. Madrid. Grupo docente procedimientos y tecnologías en Atención Primaria, 2002.

3. López Santiago A, Lara Peñaranda R, De Miguel Gómez A, Pérez López P, Ribes Martínez E. Cirugía menor en Atención Primaria: la satisfacción de los usuarios. Aten Prim 2000; 2: 61-9.

4. Guereña MJ, Perna C, Gajate J. Correlación clinicopatológica de 370 casos de cirugía menor dermatológica realizada por médicos de familia. Aten Prim 2001; 5: 320-5.

5. Arribas Blanco JM, Rodríguez Salceda I, Mena Mateo S, Martín Martín S, Bru Amantegui S, Villaroel Rodríguez J. Cirugía menor en la consulta del médico de familia. Descripción de un año de experiencia. Aten Prim 1996; 17: 142-6.

6. Pérez Unanua MP, Alonso Sacristán P, Roiz Fernández JC. Evaluación de las actividades de cirugía menor realizadas en el centro de salud. Aten Prim 2002; 12: 72-3.

7. Cox NH, Wagstaff R, Popple AW. Using clinicopathological analysis of general practitioner skin surgery to determine educational requeriments and guidelines. BMJ 1992; 304: 575.

8. Menárguez Puche JF, Alcántara Muñoz PA. Calidad de la cirugía menor realizada en Atención Primaria. ¿Son adecuados los indicadores habituales? Aten Prim 2001; 28: 80.

9. Alcántara Muñoz PA, Martínez Villalba MJ, Menárguez Puche JF, Rubio Espín A, Martínez Gonzálvez AB, Albadalejo Serrano AP. Crioterapia en Atención Primaria: eficaz y segura. Comunicación al XIX Congreso de la SEMFyC. Tenerife, diciembre de 1999.

10. Rodríguez Caravaca M, Villar del Campo M. C, González Mosquera M, Úcar Corral E, López Bran E. Comparación de la validez diagnóstica y concordancia entre Atención Primaria y Atención Especializada en Dermatología. MEDIFAM 2000; 10: 223-8. 